\title{
Estudo Comparativo de Quatro Critérios de Classificação para Artrite Psoriásica
}

\section{Comparative Study of Four Classification Criteria for Psoriatic Arthritis}

\author{
Cláudia Diniz Lopes Marques ${ }^{(1)}$, Alain Cantagrel(2) ${ }^{(2)}$ Fernando Cavalcanti $^{(3)}$, \\ Ângela Luzia Branco Pinto Duarte ${ }^{(4)}$
}

\section{RESUMO}

Objetivo: comparar o desempenho de quatro grupos de critérios propostos para definir artrite psoriásica $(\mathrm{AP})$ em pacientes portadores de artropatia inflamatória: Moll e Wright, Bennet, Vasey e Espinoza e Fournié. Métodos: foram analisados dados clínicos e laboratoriais de 195 pacientes divididos em dois grupos: 65 portadores de artrite psoriásica (grupo AP) e 130 portadores de artrite reumatóide (grupo AR). Os casos foram representados pelo grupo AP. Após definição dos falsos positivos, verdadeiros negativos, verdadeiros positivos e falsos negativos foram calculadas a sensibilidade e a especificidade de cada critério. Resultados: os critérios de Fournié foram os que apresentaram melhor desempenho, com sensibilidade de $93,84 \%$ e especificidade de $96,22 \%$. Os de Bennet foram os que demonstraram sensibilidade mais baixa $(26,15 \%)$, por outro lado, obtiveram especificidade de 100\%. Conclusão: os critérios de Fournié parecem ser os mais efetivos em identificar as diversas formas da $\mathrm{AP}$, inclusive nos casos da $\mathrm{AP}$ sem lesão cutânea ou nas formas entesopáticas difusas, permitindo que se faça diagnóstico mais precocemente e evitando as possíveis complicações que podem levar à incapacidade e deformidades permanentes.

Palavras-chave: artrite psoriásica, critérios de classificação, espondiloartropatias.

\section{INTRODUÇÃO}

Definida por Moll e Wright e outros autores ${ }^{(1-4)}$ como a associação de uma artrite inflamatória, de psoríase e de uma sorologia reumatóide geralmente negativa, a artrite psoriásica $(\mathrm{AP})$ vem demonstrando, através da experiência clínica, tratar-se de uma entidade bem mais complexa que um reumatismo inflamatório crônico em um contexto psoriásico. Apesar de guardar semelhanças com a artrite reumatóide $(\mathrm{AR})$ (por seu comprometimento periférico e de pequenas articulações) e com as espondiloartropatias,

\begin{abstract}
Objective: to compare the sensitivity and specificity of the four classification criteria of psoriatic arthritis (PA) in patients with inflammatory arthropathy: the Moll's and Wright's criteria, Bennet criteria, Vasey and Espinoza's criteria and Fournié's criteria. Methods: we analysed 195 patients distributed in two groups: 65 patients with psoriatic arthritis (PA group) and 130 patients with rheumatoid arthritis ( $R A$ group). After defining the true positives, true negatives, false positives and false negatives, we calculated the sensitivity and specificity of each criteria. Results: the Fournié's criteria were those with better performance, showing a sensitivity of $93.84 \%$ and specificity of $96.22 \%$. The Bennet's criteria had a lower sensitivity (26.15\%), but on the other hand, the high specificity (100\%). Conclusion: the Fournié's criteria appears to be the most efficient to identify the various PA's forms, including the cases of PA without cutaneous psoriasis or in the group with diffuse enthesopathies, allowing an early diagnostic and avoiding the possible complications that can lead to permanent incapacity and deformity.
\end{abstract}

Keywords: psoriatic arthritis, classification criteria, spondyloarthropathies.

grupo do qual faz parte pelo comprometimento axial, a AP vem se firmando como uma desordem distinta com uma grande variedade de formas de apresentação clínica ${ }^{(5)}$.

Calcula-se que 5 a $7 \%$ dos pacientes com psoríase vão desenvolver artrite periférica, mas esta prevalência pode chegar a $42 \%$ quando o comprometimento axial e periférico é simultâneo, enquanto na população geral esta prevalência é de 2 a $3 \%\left({ }^{(6)}\right.$.

Diversos estudos têm sugerido a associação da AP com antígenos HLA classe I - B16, B17, B27, Cw6 e os resultados têm sido controversos quanto à prevalência de

\footnotetext{
Departamento de Medicina Interna, Disciplina de Reumatologia da Universidade Federal de Pernambuco (UFPE). Recebido em 19/05/05. Apovado, após revisão, em 02/11/05.

1. Mestra em Medicina Interna pela UFPE e Doutoranda em Saúde Pública pelo Centro de Pesquisas Ageu Magalhães da Fundação Oswaldo Cruz (FIOCRUZ),

2. Professor Adjunto e Chefe do Serviço de Reumatologia do Centre Hopitalier Universitaire Rangueil, Toulouse, França.

3. Professor Adjunto da Disciplina de Reumatologia do Hospital das Clínicas da UFPE.

4. Professora Titular da Disciplina de Reumatologia do Hospital das Clínicas da UFPE.

Endereço para correspondência: Cláudia Marques, Avenida Prof. Moraes Rego, s/n, Recife, PE, Brasil, tel. (81) 3454-0155, e-mail: claudiadlmarques@terra.com.br
} 
um antígeno em determinado subgrupo da doença. São também descritas associações com antígenos da classe II - DR4 (associado com a forma poliarticular simétrica periférica) e DR7 (considerado como protetor) ${ }^{(7-12)}$.

$\mathrm{O}$ grande problema para o diagnóstico da AP aparece quando estamos diante de um paciente com quadro articular sugestivo e ausência de psoríase. Em 25\% dos casos, as lesões de pele só aparecerão após o início do quadro articular, podendo o preceder em até 15 anos $^{(13)}$, tornando neste momento o diagnóstico diferencial (principalmente das formas poliarticulares simétricas) com a AR bastante complicado. Contudo, somente o subseqüente aparecimento da psoríase pode estabelecer o diagnóstico ${ }^{(14)}$.

Devido ao perfil variado da AP, existe uma grande dificuldade em se formular o critério de classificação ideal. Apesar da ampla evidência clínico-epidemiológica mostrar se tratar de uma entidade distinta e ser reconhecida como parte do grupo das espondiloartropatias, ainda não existem critérios de classificação validados para $\mathrm{AP}^{(15)}$.

O objetivo principal do nosso trabalho foi comparar os critérios de Moll e Wright (Tabela 1)(1) com outros três grupos de critérios: Bennet (Tabela 2) ${ }^{(15)}$, Vasey e Espinoza $(\text { Tabela } 3)^{(16)}$ e Fournié (Tabela 4$)^{(17)}$, para determinar qual deles possui melhor desempenho na classificação da AP e no diagnóstico diferencial da $\mathrm{AR}$, através da avaliação da sensibilidade e especificidade de cada grupo de critérios.

TABela 1

CRITÉrios DE CLASSIFICAÇÃo PARA ARTRITE PSORIÁSICA (AP) De Moll e Wright ${ }^{(1)}$

1. Psoríase da pele ou das unhas

2. Artrite periférica ou axial

3. Fator reumatóide (FR) negativo

É necessária a presença dos três critérios

\section{MATERIAIS E MÉTODOS}

\section{SELEÇÃO DOS PACIENTES}

Foram analisados, retrospectivamente, através dos prontuários médicos, 195 pacientes portadores de artropatia inflamatória, acompanhados no Serviço de Reumatologia do Hospital Rangueil, em Toulouse, na França, no período de maio a setembro de 1999. Os pacientes foram divididos em dois grupos: o primeiro grupo constava de 65 pacientes com diagnóstico de AP (grupo AP), baseado nos seguintes critérios: 1) Presença de lesão formal de psoríase cutânea, atual ou passada, ou na ausência de psoríase pessoal, antecedentes familiares diretos de psoríase, asso- ciado a 2) um acometimento articular: dor de articulação interfalangiana distal (IFD) de mãos ou pés, ou oligoartrite periférica assimétrica, ou associada à sacroileíte ou acometimento inflamatório de coluna, ou poliartrite simétrica ou assimétrica, na ausência de fator reumatóide (FR). Única exceção foi feita a um paciente que não apresentava

Tabela 2

Critérios de ClassificaÇão para arTrite PSORIÁSICA (AP) DE BENNET ${ }^{(15)}$

$\frac{\text { Critério obrigatório: }}{\text { 1. Psoríase associada à dor e edema e/ou uma limitação da mobilidade de }}$
pelo menos uma articulação, por mais de seis semanas

Critérios secundários:

2. Dor e edema de uma ou mais articulações, constatadas por médico, por mais de seis semanas

3. Presença de sinais inflamatórios de IFD, exclusão dos nódulos de Heberden

4. Presença de dedos em "salsicha"

5. Artrites assimétricas de mãos ou pés

6. Ausência de nódulos subcutâneos

7. Ausência de fator reumatóide (FR)

8. Líquido sinovial inflamatório, ausência de infecção e de cristais de urato de sódio ou de pirofosfato de cálcio

9. Biopsia sinovial com hipertrofia e infiltrado inflamatório; ausência de granuloma ou tumor

10. Raio $X$ art. periféricas - erosão de pequenas articulações, sem osteoporose; exclui as artroses erosivas

11. Raio X axial: sacroileíte; sindesmófito; ossificação paravertebral

Definido: critério obrigatório + seis secundários

Provável: critério obrigatório + quatro secundários

Possivel: critério obrigatório + dois secundários

TABELA 3

CRITÉrios de CLASSIFICAÇÃo PARA ARTRITE PSORIÁSICA (AP) DE VASEY E EsPinOzA ${ }^{(16)}$

\begin{tabular}{c} 
Critério obrigatório \\
\hline $\begin{array}{c}\text { 1. Psoríase cutânea ou ungueal } \\
\text { 2. Dor e edema articulares das IFD durante mais de } \\
\text { quatro semanas }\end{array}$ \\
$\begin{array}{c}\text { 3. Dor e edema assimétricos das articulações periféricas durante mais de } \\
\text { quatro semanas }\end{array}$ \\
4. Artrite periférica simétrica por mais de quatro semanas, \\
na ausência de fator reumatóide (FR) \\
e de nódulos subcutâneos \\
5. Raio X periférico: osteólise em "ponta de lápis", erosão de falanges, \\
periostite irregular, anquilose óssea \\
6. Dor na coluna e limitação dos movimentos durante mais de \\
quatro semanas \\
7. Raio X axial: sacroileíte bilateral de grau 2 ou unilateral \\
de grau 3 ou 4
\end{tabular}

Rev Bras Reumatol, v. 46, n.3, p. 181-187, mai/jun, 2006 
TABELA 4

CRITÉrIOS DE CLASSIFICAÇão PARA ARTRITE PSORIÁSICA (AP) DE FourniÉ(17)

\begin{tabular}{|c|}
\hline Sinais clínicos: \\
\hline $\begin{array}{l}\text { 1. Psoríase anterior ou concomitante ao início do } \\
\text { reumatismo - } 6 \text { pontos }\end{array}$ \\
\hline $\begin{array}{l}\text { 2. Psoríase familiar na ausência de psoríase pessoal ou psoríase } \\
\text { posterior ao início do reumatismo - } 3 \text { pontos }\end{array}$ \\
\hline 3. Artrite de uma IFD - 3 pontos \\
\hline 4. Acometimento cérvico - dorsal - 3 pontos \\
\hline 5. Mono ou oligoartrite assimétrica - 1 ponto \\
\hline $\begin{array}{l}\text { 6. Dor inflamatória em calcanhares, nádegas, esternocostoclavicular } \\
\text { ou entesalgias difusas sensíveis aos AINH } \\
\text { (antiinflamatórios não-hormonais) - } 1 \text { ponto }\end{array}$ \\
\hline Sinais radiológicos: \\
\hline 7. Um dos critérios radiológicos de Ávila - 5 pontos \\
\hline Laboratório: \\
\hline 8. Presença do antígeno HLA B16 $(38,39)$ ou B17 - 4 pontos \\
\hline 9. Ausência de fator reumatóide (FR) - 4 pontos \\
\hline $\begin{array}{c}\text { Se a soma dos pontos é } \geq 11, \text { o paciente será classificado como } \\
\text { portador de AP }\end{array}$ \\
\hline
\end{tabular}

psoríase, diagnosticado "AP sem lesão cutânea" pelo médico acompanhante, devido ao quadro clínico extremamente sugestivo: acometimento de IFDs e coluna, com presença de sindesmófitos; oligoartrite assimétrica e FR negativo. Foi considerado como portador de psoríase o paciente com lesão cutânea constatada por um médico clínico, e no caso de dúvida, confirmado por dermatologista. $\mathrm{O}$ segundo grupo era representado por 130 pacientes portadores de AR (grupo AR), diagnosticados de acordo com os critérios do American College of Rheumatology (ACR) ${ }^{(18)}$. Os grupos foram pareados por idade, numa relação de dois pacientes do grupo AR para um do grupo AP.

Os pacientes de ambos os grupos possuíam radiografias das articulações afetadas, incluindo mãos, pés, coluna e sacroilíacas. A análise laboratorial incluiu a pesquisa de FR de classe IgM, por Enzyme-Linked Immunosorbent Assay (ELISA); anticorpos antinucleares (FAN) por imunofluorescência pela técnica HEp2; avaliação da síndrome inflamatória (VHS - velocidade de hemossedimentação e PCR - proteína C reativa) e tipagem HLA de classe I e II.

\section{CRITÉRIOS CLASSIFICATÓRIOS}

Os pacientes dos dois grupos foram submetidos aos seguintes critérios: Moll e Wright ${ }^{(1)}$, Bennet ${ }^{(15)}$, Vasey e Espinoza ${ }^{(16)}$ e Fournié(17). No caso dos critérios de Bennet, levamos em consideração apenas a definição de caso “definido" (Tabela 2). Os critérios radiológicos utilizados foram os de Ávila modificado ${ }^{(19)}$, que graduam as alterações radiológicas numa escala que varia de 1 a $5: 1$ ) artrite erosiva de IFD; 2) deformidade "lápis na taça"; 3 ) anquilose da articulação interfalangiana; 4) periostite justa-articular em uma MTF; 5) osteoperiostite ou acroosteólise do hálux. Foram também testados os critérios do $\mathrm{ACR}^{(18)} \mathrm{em}$ todos os pacientes do grupo AP.

\section{ANÁLISE ESTATÍSTICA}

Após definição dos falsos positivos, verdadeiros positivos, falsos negativos e verdadeiros negativos, foram calculados a sensibilidade, especificidade, valor preditivo positivo e valor preditivo negativo de cada um dos critérios, seguindo as fórmulas usuais.

\section{RESULTADOS}

A Tabela 5 resume os dados clínico-epidemiológicolaboratoriais nos dois grupos. No grupo AP, não houve diferença significante em relação ao sexo $(44,7 \%$ eram do sexo masculino e $55,3 \%$, do sexo feminino). Cinqüenta e nove pacientes apresentavam psoríase $(90,8 \%)$ (Tabela 5$)$, sendo que a maioria $(41 ; 69,49 \%)$ apresentava a psoríase vulgar, considerada a forma clássica da doença cutânea.

Em um caso, as lesões de pele apareceram após o início do quadro articular (1,69\%). Seis pacientes não apresentavam psoríase $(9,2 \%)$, porém cinco deles tinham antecedentes familiares diretos da doença $(7,69 \%)$. O outro paciente foi considerado como "AP sem psoríase". Interessante notar que apesar de serem evidenciados sindesmófitos em 10,7\% dos pacientes, apenas 3,07\% apresentava sintomas de envolvimento inflamatório da coluna, sendo apenas este grupo considerado como portador da forma axial da doença.

No grupo AR, houve predominância do sexo feminino $(63,84 \%)$. Neste grupo, oito pacientes apresentavam psoríase e quatro pacientes tinham antecedentes familiares diretos da doença $(3,07 \%)$. Encontramos uma maior associação de HLA DR7 (36,9\%) e Bl6 (24,6\%) no grupo AP.

\section{COMPARAÇÃO DOS QUATRO CRITÉRIOS DE CLASSIFICAÇÃO}

A Tabela 6 mostra a comparação entre a sensibilidade, especificidade, valor preditivo positivo e valor preditivo negativo dos quatro grupos de critérios. Vale salientar que apenas três pacientes do grupo AP preencheram os critérios do ACR para AR (4,3\%).

Dentro da população estudada, os critérios de Fournié foram os que apresentaram índice de sensibilidade mais 
TABELA 5

DADOS CLÍNICO-EPIDEMIOLÓGICO-LABORATORIAIS NOS DOIS GRUPOS

\begin{tabular}{|c|c|c|}
\hline & Artrite psoriásica AP $(n=65)$ & Artrite Reumatóide (AR) $(n=130)$ \\
\hline Média de idade (anos) & $44,8(25-82)$ & $46,9(21-83)$ \\
\hline Sexo $(M / F)$ & $36 / 29(44,7 \% / 55,3 \%)$ & $49 / 83(36,1 \% / 63,8 \%)$ \\
\hline Duração média de evolução da artropatia (meses) & 15,2 & 15,5 \\
\hline Artrite assimétrica & $60(92,3 \%)$ & $7(5,3 \%)$ \\
\hline Artrite simétrica & $3(4,6 \%)$ & $123(94,6 \%)$ \\
\hline Acometimento de IFD & $20(30,7 \%)$ & $5(3,8 \%)$ \\
\hline Entesalgia, acometimento esternocostoclavicular & $50(76,9 \%)$ & $9(6,9 \%)$ \\
\hline Acometimento cérvico-dorsal inflamatório & $33(50,7 \%)$ & $18(13,8 \%)$ \\
\hline Dedos em "salsicha" & $13(20 \%)$ & $1(0,7 \%)$ \\
\hline Forma axial & $2(3,07 \%)$ & 0 \\
\hline Nódulos & 0 & $3(2,3 \%)$ \\
\hline Sacroiliíte & $4(6,1 \%)$ & 0 \\
\hline Alts. Radiológicas de pequenas articulações & $14(21,5 \%)$ & $38(29,2 \%)$ \\
\hline Critérios de Ávila & $6(9,2 \%)$ & 0 \\
\hline Sindesmófitos & $7(10,7 \%)$ & $1(0,7 \%)$ \\
\hline Líquido Inflamatório & $14(21,5 \%)$ & $17(13,07 \%)$ \\
\hline Psoríase & $59(90,8 \%)$ & $8(6,1 \%)$ \\
\hline Vulgar & $41(69,5 \%)$ & $8(6,1 \%)$ \\
\hline Ungueal & $5(8,5 \%)$ & 0 \\
\hline Pustulosa & $2(3,3 \%)$ & 0 \\
\hline Couro cabeludo & $21(35,6 \%)$ & 0 \\
\hline Familiar & $5(7,6 \%)$ & $4(3,07 \%)$ \\
\hline Posterior & $1(1,5 \%)$ & 0 \\
\hline VHS - velocidade de hemossedimentação & 10,7 & 24,6 \\
\hline PCR - proteína $C$ reativa & 18,4 & 32,3 \\
\hline FR - fator reumatóide & 1,5 & 76,9 \\
\hline FAN - anticorpos antinucleares & 20,0 & 43,8 \\
\hline HLA B13 & 1,5 & 2,3 \\
\hline HLA B16 & 24,6 & 7,6 \\
\hline HLA B17 & 6,1 & 5,3 \\
\hline HLA B27 & 20,0 & 6,1 \\
\hline HLA DR1 & 12,3 & 23,0 \\
\hline HLA DR4 & 13,8 & 52,3 \\
\hline HLA DR7 & 36,9 & 20,0 \\
\hline
\end{tabular}

TABELA 6

COMPARAÇÃO DA SENSIBILIDADE E ESPECIFICIDADE DOS 4 GRUPOS DE CRITÉRIOS PARA ARTRITE PSORIÁSICA (AP), APLICADOS PARA PACIENTES PORTADORES DE AP E ARTRITE REUMATÓIDE (AR)

\begin{tabular}{ccc|c|c}
\hline & Moll e Wright & Bennet & Vasey e Espinoza & Fournier \\
\hline Falsos positivos & 3 & 0 & 5 & 4 \\
\hline Verdadeiros positivos & 58 & 17 & 59 & 61 \\
Falsos negativos & 7 & 48 & 125 & 126 \\
Verdadeiros negativos & 127 & 130 & 92,18 & 93,84 \\
VPP (\%) & 95,08 & 100 & 95,41 & 96,92 \\
VPN (\%) & 94,77 & 73,03 & 90,76 & 93,84 \\
\hline Sensibilidade (\%) & 89,23 & 100 & 96,15 & 96,92 \\
\hline Especificidade (\%) & 97,69 & & & \\
\hline
\end{tabular}

VPP: Valor preditivo positivo; VPN : Valor preditivo negativo. 
elevado $(93,84 \%)$ e os de Bennet “definido", a mais baixa. O desempenho dos quatro grupos de critérios com relação à especificidade foi bastante semelhante, sendo a de Bennet a mais elevada $(100 \%)$.

\section{DISCUSSÃO}

A AP só foi reconhecida como uma entidade distinta da AR, incluída dentro do grupo das espondiloartropatias soronegativas em 1959, após a descrição de Wright da freqüente associação de psoríase cutânea e envolvimento das articulações IFDs com erosão e reabsorção das falanges terminais, coexistindo com sacroileíte, comprometimento das interfalangianas proximais (IFPs) dos pés e artrite mutilante. Apesar desse reconhecimento, ainda existem controvérsias sobre quais pacientes devem ser incluídos neste grupo, uma vez que, devido a algumas dificuldades metodológicas impostas pela doença (amplo espectro clínico, o curso geralmente intermitente e a baixa incidência na população geral $)^{(5)}$, ainda não dispomos de critérios de classificação específicos validados e aceitos pela comunidade científica ${ }^{(20)}$.

Os critérios mais freqüentemente utilizados em estudos clínico-epidemiológicos são os de Moll e Wright ${ }^{(1)}$, que classifica todo paciente portador de psoríase associada a uma artrite inflamatória (incluindo pacientes com doença leve, envolvimento espinhal e monoartrite) e sorologia reumatóide geralmente negativa. Salvarani et a ${ }^{(8)}$ estudaram a prevalência da AP em pacientes italianos utilizando os critérios de Moll e Wright ${ }^{(1)}$ e encontraram uma sensibilidade de $61 \%$ e uma especificidade de $100 \%$. Já Fournié et al ${ }^{17}$ encontraram em seu estudo sensibilidade de $70 \%$ e especificidade de $97 \%$ para estes mesmos critérios. Os critérios de Moll e Wright apresentam, portanto, boa especificidade, mas sensibilidade baixa. Além do mais, são restritivos e inadequados para definir pacientes psoriásicos com entesite e/ou dactilite e excluem os pacientes sem psoríase ou com apenas antecedentes familiares da doença.

Em 1979, Bennet et al ${ }^{15)}$ propuseram uma revisão dos critérios de classificação inspirando-se na definição de Moll e Wright de 1973. Estes incluem um critério obrigatório, definido como a presença de psoríase com envolvimento de pele ou unha, em associação com dor e/ou edema de partes moles e/ou limitação da mobilidade em pelo menos uma articulação, observada por médico, por pelo menos seis semanas, associado a dez critérios secundários (Tabela 2). Se seis dos dez critérios secundários estiverem presentes, pode-se fazer o diagnóstico de caso "definido"; se quatro estiverem presentes, caso "provável", e se dois estiverem presentes, AP “possível”. Estes critérios são baseados na definição de AP e nas características clínicas geralmente observadas nesta patologia. Contudo, ainda não foram testados para confirmar sua validade.

Os critérios propostos por Vasey e Espinoza em 1990(16) também apresentam um critério obrigatório, definido como a presença de psoríase cutânea ou ungueal, associada a dois outros critérios principais que incluem artrite periférica e comprometimento axial e levam em consideração os padrões radiológicos característicos da $\mathrm{AP}$, como periostite, reabsorção dos tufos terminais, deformidade "lápis na taça" e anquilose óssea. O diagnóstico pode ser feito se o critério obrigatório está presente, associado a qualquer dos subitens dos dois outros critérios principais. Esses critérios também ainda não foram validados ${ }^{(21)}$.

Tanto os critérios de Bennet ${ }^{(15)}$ como os de Vasey e Espinoza $^{(16)}$ não têm sido muito difundidos na prática clínica $^{(4)}$ e, assim como os de Moll e Wright ${ }^{(1)}$, falham em não reconhecer o paciente com quadro articular $\mathrm{e}$ sem psoríase, pois a presença de lesão cutânea é critério mandatório. Isto induz a se pensar que toda artrite inflamatória que aparece num paciente portador de psoríase é uma AP, quando se sabe que ela pode acompanhar outras artropatias inflamatórias crônicas, ou que, por outro lado, a presença de psoríase é indispensável ao diagnóstico ${ }^{(18,21)}$. Diversos estudos ${ }^{(9,22-25)}$ já demonstraram sinais extremamente sugestivos de AP em pacientes sem psoríase, ou mesmo antecedentes familiares da doença.

Em 1999, Fournié et al ${ }^{(17)}$ propuseram uma nova forma de classificação, diferente do que já havia sido publicado anteriormente. Essa se baseia em três critérios principais, sendo eles clínicos, radiológicos e biológicos, e cada sub-item recebe um coeficiente de ponderação diferente, sendo o coeficiente do item "presença de psoríase" o mais elevado (igual a seis). O diagnóstico é feito se a soma dos critérios positivos for maior ou igual a 11 . O fato de não apresentar como critério "obrigatório" a presença de psoríase, permite que ela classifique pacientes portadores de AP "sem lesão cutânea" e, além disso, permite identificar os vários aspectos clínicos da $\mathrm{AP}$, incluindo as formas entesopáticas difusas.

Em nosso estudo, testamos estes quatro grupos de critérios em pacientes portadores de artrite inflamatória inicial, tendo como objetivo saber se eles permitem distinguir AR e AP nas fases iniciais da doença. Os critérios de Fournié et a ${ }^{17)}$ foram os que apresentaram o melhor desempenho, com sensibilidade de $93,84 \%$ e especificidade próxima a dos outros critérios $(96,92 \%)$ (Tabela 6), 
resultados esses semelhantes ao trabalho dos autores $(95 \mathrm{e}$ $98 \%$ respectivamente $)^{(17)}$. Deve-se notar também que somente seis pacientes tiveram os critérios radiológicos de Ávila presentes e que, para estes mesmos pacientes, o diagnóstico não seria modificado se nós ignorássemos estes critérios. Os critérios de Ávila são, portanto, pouco úteis ao diagnóstico precoce da AP. A presença de quatro falsos positivos deu um valor preditivo positivo $92,18 \%$.

O desempenho dos critérios de Moll e Wright ${ }^{(1)}$ e Vasey e Espinoza ${ }^{(16)}$ foram equiparáveis (sensibilidade de $89,23 \%$ e especificidade de $97,69 \%$ para os de Moll e Wright $^{(1)} ; 90,76 \%$ de sensibilidade e $96,15 \%$ para os de Vasey e Espinoza). Por outro lado, os critérios de Moll e Wright ${ }^{(1)}$ são os que apresentam melhor valor preditivo positivo $(95,08 \%)$. Nestas duas séries de critérios, os falsos negativos são diretamente ligados aos pacientes portadores de AP sem doença cutânea, mostrando suas limitações em reconhecer esta variável da doença.

Utilizando os critérios de Bennet ${ }^{(15)}$, obtivemos uma sensibilidade extremamente baixa $(26,15 \%)$ nos casos "definidos", com 48 falsos negativos, dando um valor preditivo positivo de $73,03 \%$. Isso se deve à presença de critérios com "biópsia sinovial inflamatória" ou "líquido articular inflamatório", uma vez que nem todos os pacientes são submetidos a tais procedimentos considerados invasivos, principalmente no início do quadro articular inflamatório. Por outro lado, a especificidade desses critérios foi a mais elevada, atingindo $100 \%$, com valor preditivo positivo equivalente também de $100 \%$.

Além do grupo de critérios estudados, existem outros descritos na literatura, com os de $\mathrm{McGonagle}^{(26)}$ e os do ESSG $^{(27)}$ (European Spondyloarthropathy Study Group), porém nenhum deles validado. $\mathrm{O}$ desenvolvimento de novas terapias, particularmente o uso de agentes biológicos, tem reforçado esta deficiência e demonstrado a necessidade urgente de estudos para validação destes critérios e para

\section{REFERÊNCIAS}

1. Moll JMH, Wright TV: Psoriatic arthritis. Semin Arthritis Rheum 3: 55-78, 1973.

2. Gladman DD: Psoriatic arthritis recent advances in pathogenesis and treatment. Rheum Dis Clin North Am 18: 247-56, 1992.

3. Scarpa R, Oriente P, Pucino A: Psoriatic arthritis in psoriatic patients. Br J Rheumatol 23: 246-50, 1984

4. Veale D, Rogers S, Fitzgerald O: Classification of clinical subsets in psoriatic arthritis. Br J Rheumatol 33: 133-8, 1994.

5. Gladman DD: Natural history of psoriatic arthritis. Bailliere's Clin Rheumatol 8: 379-393, 1994.

6. O’Neill T, Silman AJ: Psoriatic Arthritis: Historical background and uma padronização de critérios de resposta e de evolução. O problema maior desta padronização não está na forma clássica de apresentação da doença - oligoartrite com comprometimento de IFDs, entesite e dactilite -, mas naquela onde temos poliartrite soronegativa e psoríase ${ }^{(21)}$.

\section{CONCLUSÃO}

Os critérios propostos por Fournié(17) parecem ser os mais efetivos em identificar as diversas formas clínicas da $\mathrm{AP}$, incluindo as formas entesopáticas difusas e AP "sem lesão cutânea”, mesmo nos casos de artropatia inflamatória aguda. Leva vantagem sobre os outros critérios pela não obrigatoriedade da presença de psoríase. Vale salientar que os valores obtidos na sensibilidade, especificidade, valor preditivo positivo e negativo referem-se à comparação de critérios de AP com um critério de classificação para $\mathrm{AR}$, já que o nosso objetivo era tentar classificar pacientes com quadro de artrite inicial, principalmente aqueles que não apresentam a psoríase cutânea.

Contudo, estudos futuros serão necessários para confirmar esses achados e validar os critérios, tornando-os úteis na prática, quando o quadro clínico-laboratorial não é típico, fazendo o diagnóstico mais precocemente, permitindo instituição de tratamento adequado e evitando as possíveis complicações. Apesar de a maioria dos pacientes apresentar a artrite associada à psoríase de evolução benigna, outros podem evoluir de forma rápida e brutal, levando à incapacidade e deformidades permanentes.

\section{AGRADECIMENTOS}

Agradecemos ao Serviço de Reumatologia do Centre Hopitalier Universitaire Rangueil em Toulouse, na França, na pessoa dos professores Alain Cantagrel e Bernard Maziéres, pela oportunidade do estágio e realização deste trabalho.

epidemiology. Bailliere's Clin Rheumatol 8: 245-260, 1994.

7. Gladman DD, Anhorn KAB, Schachter RK, Mervat H: HLA antigens in psoriatic arthritis. J Rheumatol 13: 586-92, 1986.

8. Salvarani C, Lo Scocco G, Macchioni P et al: Prevalence of psoriatic arthritis in italian psoriatic patients. J Rheumatol 22: 1499-1502, 1995.

9. Founié B, Granel J, Heraud A et al: HLA B et rhumatisme psoriasique Etude prospective de 193 cas. Rev Rhum 58: 269-79, 1991

10. Estmond CJ, Woodrow JC: The HLA system and the arthropathies associated with psoriasis. Ann Rheum Dis 36: 112-20, 1997. 
11. Eastmond CJ: Genetics and HLA antigens. Bailliere's Clin Rheumatol 8: 263-74, 1994.

12. Gladman DD, Farewell VT: The role of HLA antigens as indicators of disease progression in psoriatic arthritis Multivariate relative risk model. Arthritis Rheum 38: 845-50, 1995.

13. Pitzalis C: Skin and joint disease in psoriaitc arthritis what's the link? Br J Rheumatol 37: 480-3, 1998.

14. Gladman DD: Psoriatic arthritis. Bailliere's Clin Rheumatol 9: 319-27, 1995.

15. Bennet RM Psoriatic Arthritis. In: McCarty DJ (ed). Arthritis and Related Conditions Philadelphia Lea \& Febiger, 645, 1979.

16. Vasey LB, Espinoza LR. Psoriatic arthritis. In Calin A (ed). Spondyloarthropathies. Rev Rheum 57: 85-9, 1990.

17. Fournié B et al: Proposed classification criteria of psoriatic arthritis A preliminary study in 260 patients. Rev Rheum (Engl Ed) 66: 446-56, 1999.

18. Arnett FC, Edworthy SM, Bloch DA et al: The American Rheumatism Association 1987 revised criteria for the classification of rheumatoid arthritis. Arthritis Rheum 31: 315-24, 1988.

19. Avila R, Pugh DG, Slocum CH, Winkelmann RK: Psoriatic arthritis: a roentgenologic study. Radiology 75: 691-702, 1960.

20. Helliwell PS, Taylor WJ: Classification and diagnostic criteria for psoriatic arthritis. Ann Rheum Dis 64 (Suppl II): ii3-ii8, 2005.

21. Salvarani C, Cantini F, Olivieri I et a: Isolated peripheral enthesitis and/or dactylitis a subset of psoriatic arthritis. J Rheumatol 24: 1106-10, 1997.

22. Marsal S, Armandans-Gil L, Martinez M, Gallardo D, Ribera A, Lience E: Clinical, radiographic and HLA associations as markers for different patterns of psoriatic arthritis. Rheumatol 38: 332-7, 1999.

23. Green L, Meyers OL, Gordon W, Briggs B: Arthritis in psoriasis. Ann Rheum Dis 40: 366-9, 1981.

24. Tarkowski A, Nilson LA: Rheumatoid factors in psoriatic arthropaty and in Waaler Rose negative rheumatoid arthritis. Rheumatol Int 4:115-117, 1984.

25. Fournié A, Ayrolles C, Sellami S, Guyard C, Fournié B: Histoire naturelle du rhumatisme psoriasique. Etude prospective sur 82 cas. Rev Rhum 47: 309-16, 1980.

26. McGonagle D, Conaghan PG, Emery P: Psoriatic arthritis a unified concept twenty years on. Arthritis Rheum 42: 1080-1086, 1999.

27. Dougados M, van der Linden S, Juhlin R et al: The European Spondyloarthropathy Study Group preliminary criteria for the classification of spondyloarthropaty. Arthritis Rheum 34: 121827,1991 . 